\title{
The role of orthotics in the rehabilitation of patients with fracture of the calcaneum
}

\author{
W. WINKLER \\ SUVA Rehabilitation Clinic, Bellikon, Switzerland
}

\section{Introduction}

Fractures of the calcaneum comprise about $1-2 \%$ of all fractures and as such are not common, yet in the author's experience they are surprisingly frequent.

Regrettably the various classifications offered by Essex-Lopresti (1952), Judet (1954), Rowe (1963), Böhler (1977), Vidal (1983), etc. do not lead to clear indications regarding final treatment, duration of disability or more indirectly the level of pension.

In the author's study there was a relationship between fractures involving the calcaneocuboid joint and protracted rehabilitation, an increased frequency of prescription of orthopaedic shoes and the size of the pension awarded. Although fractures with involvement of the midtarsal joint are usually mentioned in planning treatment, the late sequelae are barely discussed in the literature. Mattel's (1983) investigation on the treatment and rehabilitation of patients with fractures of the calcaneum consisted of a review of 111 cases which by the very nature of the clinic (Bellikon, Switzerland) was pre-selected. It is therefore clearly of interest to compare the results of this study with a randomly selected group of patients; specifically to compare the results in patients with a fracture of the calcaneum involving the midtarsal joint with those without involvement of the midtarsal joint. As an introduction to this topic the following statistical data is presented.

\section{Statistical data}

Various authors report a relatively high percentage of fractures without any involvement of the joint; Böhler (1977) reported $38.5 \%$. In this series the percentage without subtalar involvement was $17.6 \%$; in the author's view this incidence is still high and

All correspondence to be addressed to Dr. W. Winkler, SUVA Rehabilitation Clinic, CH-5454 Bellikon, Switzerland. serves to support the view that the classifications of these fractures are not accurate with regard to prognosis.

About one third of the patients had involvement of the lateral midtarsal joint viz., the calcaneo-cuboid. This group of patients also most frequently needed rocker soles or orthopaedic shoes and ended up with the highest pensions $(40-50 \%$ disability with unilateral calcaneal fracture involving the midtarsal joint; in contrast the average degree of disability in selected patients with unilateral calcaneal fractures is $20-25 \%$ ).

More than half of the patients resumed work within 5 to 9 months after the accident. Yet the total average duration of incapacity was 9.5 months, which shows that a small number of cases with disproportionately great problems can worsen these numbers considerably.

Arthrodesis of the "lower ankle joint" was required by $28 \%$ of all patients (lower ankle joint: the composite of the talo-navicular, talocalcaneal and calcaneo-cuboid joints). Again, this very high percentage reflects the selection of patients.

Surgical treatment of their fracture was carried out on $15 \%$ of the inpatients. As this presumably reflects the general frequency of surgical treatment of fractures in Switzerland no conclusions can be drawn regarding any special tendency.

\section{Pathology}

Orthotic management may also have its place in the acute treatment of fractures. The splint shoe with rocker bar (Fig. 1) was originally intended to replace a walking cast. Two or three different models of patellar-tendonbearing orthoses could also be used: the rather unpopular Allgower orthosis with a stirrup as the only bearing area, the Sarmiento orthosis made of thermoplastics, or a rigid orthosis made of resin with a complete sole for footrest and heel-relief. 


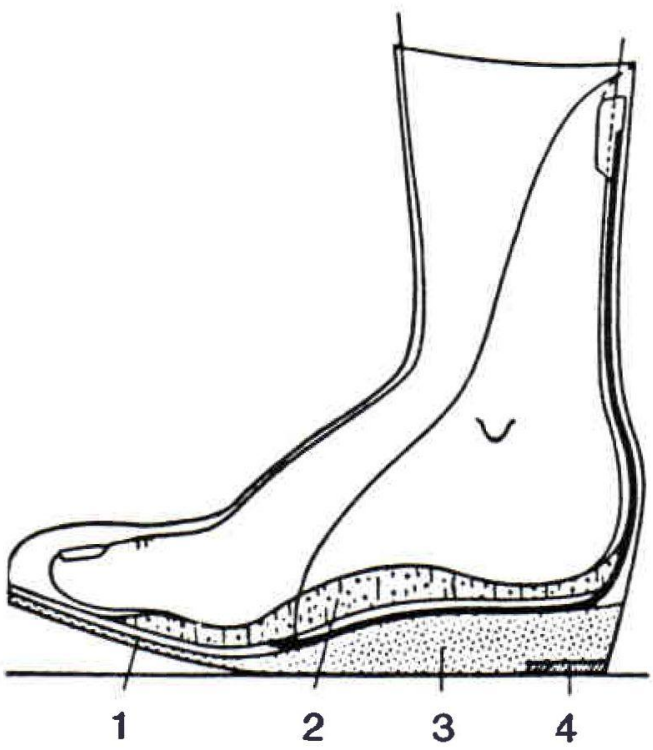

Fig. 1. Splint shoe with rocker bar by R. H. Rabl (1975). 1, bottom filler and insole; 2, inside cork insole; 3 , Airolux; 4, rigid plate.

Instead of discussing these attention should be focussed on orthopaedic measures intended to alleviate the sequelae of fractures that have already healed. Therefore in discussing the damage suffered the final condition after treatment will be considered and not the mechanisms or classifications of fractures.

\section{Morphological changes found subsequent to healing of fractures}

1. Calcaneal spur : due to displacement of bone fragments a single piece of sharp bone may present as the lowest point of the heel (Fig. 2, 1).

2. Incongruity of the subtalar joint: according to Böhler (1977) $62 \%$ of all heel fractures produce distortion within the subtalar joint. It is very rarely possible to completely correct displacement; in fact, if visible involvement of the subtalar joint is at all present incongruity must be expected as a rule. Furthermore, well preserved mobility despite incongruity of the subtalar joint may cause more pain than complete loss of mobility.

3. Reduced height of the whole foot: it is important not to consider the reduction of height as only a matter of the calcaneus. Although phylogenetically the first to third metatarsal rays belong to the talus and the fourth and fifth to the calcaneum, in terms of function it is more useful to view all the bones of the foot with exception of the talus as a single unit, which is styled the "subtalar plate". A better understanding of displacements of the whole foot, not just the heel, can be derived from this concept.

4. Dorsal tilt of the talus: this is a consequence of the collapsed "angle of Böhler" (Böhler, 1977). Frequently the author observes a changed "mid-position" of the talus which has been forced to adapt to the altered condition of the subtalar plate (Fig. 2, 4).

5. Widening of the calcaneum: since the heel is usually slightly supinated at heel strike widening is generally towards the lateral side as a result of the progression of primary and secondary fracture lines under axial stress causing outward shift of the lateral wall (Fig. 2, 6).

6. Varus or valgus deformities: they also are due to the varus position of the heel upon impact, and in most cases a varus deflection does result. Other types of fractures or severely compounded fractures may cause such a
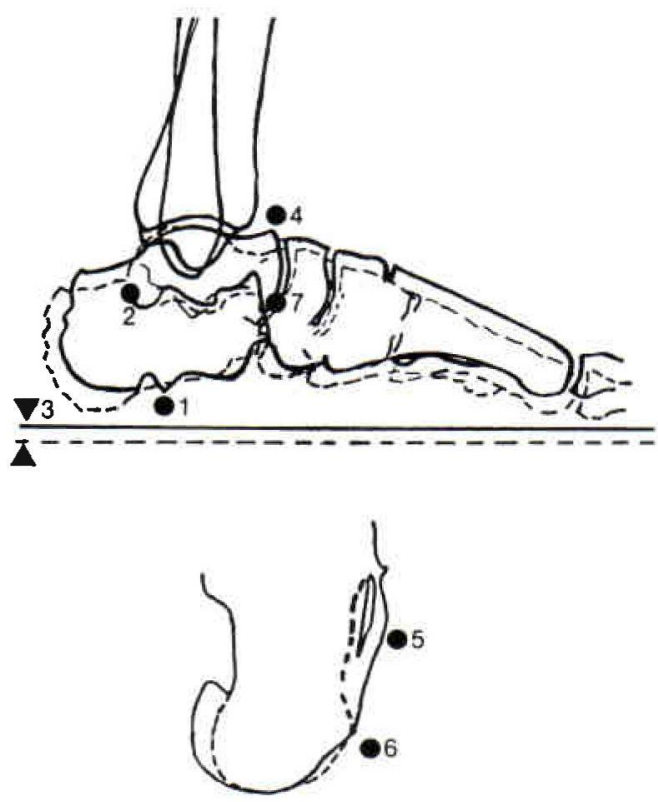

Fig. 2. Top, tracing of an X-ray of a standing foot after consolidation of a calcaneal fracture. The analogous X-ray of the contra-lateral foot is superimposed. Bottom, tracing of an axial X-ray of the hindfoot after consolidation of a calcaneal fracture. The numbers correspond with the possible permanent sequelae mentioned in the text. 
massive lateral shift of bone that a valgus deformity results secondary to floor reaction forces (Fig. 2,6).

7. Axial deviation in the midtarsal joint: this can have its greatest consequences in the talonavicular joint despite only slight displacement in the calcaneocuboid joint, or deviation may be confined mainly to the calcaneocuboid joint. The subtalar plate is weakened at this point. (Fig. 2, 7).

\section{Functional changes subsequent \\ to healed fractures}

1. Impaired muscular balance on the movements of the ankle joint proper: positional changes of the muscular attachments have taken place. The tuber calcanei has moved considerably higher and the insertion of the Achilles tendon is closer to the belly of triceps surae. The tibialis anterior, a powerful dorsiflexor of the foot, fares similarly because of the increased dorsiflexed attitude of the talus compressed as it is into the deformed calcaneum. The effective lengthening of both tendons results in weakening of the contractile forces. In particular "push off" is affected, often severely so. The author's observations here are unequivocal: even after years the muscles fail to shorten enough to re-establish normal tension. This requires particular emphasis as the triceps surae is a tonic muscle that does tend to shorten, yet apparently it cannot shorten to a significant extent without having suffered injury itself. The effects on tibialis anterior are less serious. However these muscles should not be viewed separately. What is essential is the proprioceptive control of actions by antagonistic contraction. This is frequently impaired in sequelae of calcaneal fractures and in the author's observations represents by far the most important impairment.

2. Impaired muscular function on the "lower ankle joint": this is caused in part by incongruity and therefore by morphological factors and in part by dysfunction of the peroneal tendons. Widening of the calcaneum frequently causes, by impingement, marked distortion of the common sheath for peroneus longus and brevis. The pain upon tensing of these tendons leads to reflexive release. Thus the foot, which normally proceeds through pronation and resupination during unwinding of the step (Subotnik, 1975), cannot be actively stabilized.

Secondary to the new morphological features, mobility of the "lower ankle joint" may be markedly or even almost completely limited. Instability of the "lower ankle joint" during the stance phase causes pain: active muscular stabilization could control it. Hence dysfunction of the peroneal tendons is a double hindrance: adequate adaptation of the foot to the ground surface is prevented, and sufficient stabilization of the foot to prevent painful movements becomes impossible.

3. Weakening of the subtalar plate: in normal locomotion during toe-off the subtalar plate acts as the long arm of a lever as during resupination with tensing of the plantar aponeurosis, the individual bony links of the plate become interlocked, thus preventing bending forces. Following calcaneal fractures this can be impaired in two ways. On the one hand the resultant force in the subtalar joint upon interlocking of the subtalar plate causes pain by acting on incongruent joint surfaces. On the other hand the subtalar plate may not be able to interlock and in turn is subjected to bending forces at its weakest point viz., the midtarsal joint. One can imagine the severity of impairment should this joint also have incongruent surfaces secondary to the fracture.

\section{Mechanical orthopaedic aids and their effects}

In planning technical aids it is important to keep in mind the morphological as well as the functional changes. For instance elevation of the heel with cushioning of a calcaneal spur may prevent the creation of a sufficiently flat bedding for the foot because of insufficient contractility of the crural muscles. With some experience this can be anticipated but ultimately these unintentional reciprocal effects can never be fully avoided. This must be discussed with the patient, preferably before the first fitting, for he will not readily understand why he first gets his heel elevated and later lowered again. Consequently it is necessary to emphasize discussion with the patient as the cornerstone of technical orthopaedic management.

A further important aspect is that the desired effect on the foot cannot be achieved with a loose insole or with a modified shoe on its own but usually requires an insole as well as a 
modification of the shoe. A painful midtarsal joint generally requires a rigid support. This may be attained with a removable rigid insole or with a cork and leather insole secured to the shank of a shoe which has a rigid sole or resting directly on a full thickness cradle in a custombuilt shoe.

Insole (support):

Its purpose is to distribute weight and to bridge points of weakness (Fig. 3). It is frequently employed after calcaneal fractures and is indispensable when varus deformity of the hindfoot is present and when the midtarsal joint is involved. Rather than correct a deformity the insole must be adapted to the existing shape of the foot when weightbearing. There is no exception to this rule even when a pronating correction is attempted. A support that is higher on the lateral aspect than on the medial will cause pain. However there is some justification for supinating corrections if relief of the peroneal tendons is desired as supination of the heel will give the tendons a bit more room; the support should be elevated about $2 \mathrm{~mm}$ medially above the "mid-position" of the foot.

The first metatarsal ray is more yielding to pressure from the floor than the fifth. If mobility of the "lower ankle joint" is limited, even a plantigrade metatarsus and forefoot fails to distribute the load evenly between the first and fifth metatarsal heads, and the fifth metatarsal ray becomes overloaded. This should be taken into consideration while

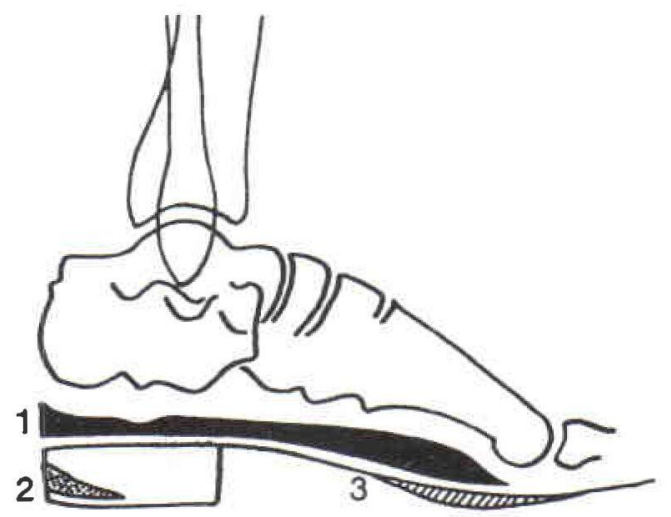

Fig. 3. Schematic representation of an orthopaedic aid for fracture of the calcaneum. 1. Insole with recess for calcaneal spur. 2. Buffer heel for shock absorption and to improve roll-over of the heel. 3 . Rocker sole: in this case it is placed behind the first metatarsal head tapering off towards the forefoot. manufacturing the insole by bedding the medial metatarsal-phalangeal joint a bit higher. Should a supination deformity of the hindfoot already be present it may even be advantageous to extend the support under the big toe, since an elevation of the first metatarsal head without simultaneous elevation of the big toe will result in a hallux flexus.

The insole is usually made from material of medium firmness or of a combination of materials. Especially rigid or hard material is recommended for pain in the midtarsal joint. Especially soft material is recommended for calcaneal spurs, but at the same time it must be taken into account that unintentional rocking on bouncy materials can cause pain in the "lower ankle joint".

\section{Buffer heel}

This works by shock absorption and by anterior displacement of the roll-over point of the heel (Figs. 3 and 4, top). With varus deformity present the buffer should be set at the middle or slightly medial to the middle of the heel. If it is applied at the usual roll-over point slightly lateral of the middle the varus deformity will be increased.

\section{Heel raise}

This affects the position of the talus and in certain cases will facilitate walking on uneven ground, especially uphill walking.

\section{Lowering of the heel}

This increases tension in the Achilles tendon and usually feels comfortable to the patient. Often patients appear in the clinic already wearing ready-made shoes with particularly low heels.

\section{Rocker sole}

By moving the roll-over point back a smaller range of motion is required in the true ankle joint and less load is transferred to the forefoot during toe-off (Figs. 3 and 4, centre and bottom). Rocker soles or roller bars additionally have a similar effect to lowering the heel. Thus if the position of the foot becomes too dorsiflexed the rocker must be accompanied by an elevation of the heel.

In addition to the height of the rocker at its crest and the position of the crest in relation to the foot it is possible to vary the position in relation to the direction of roll-over. Usually the crest is exactly at right angles to the 

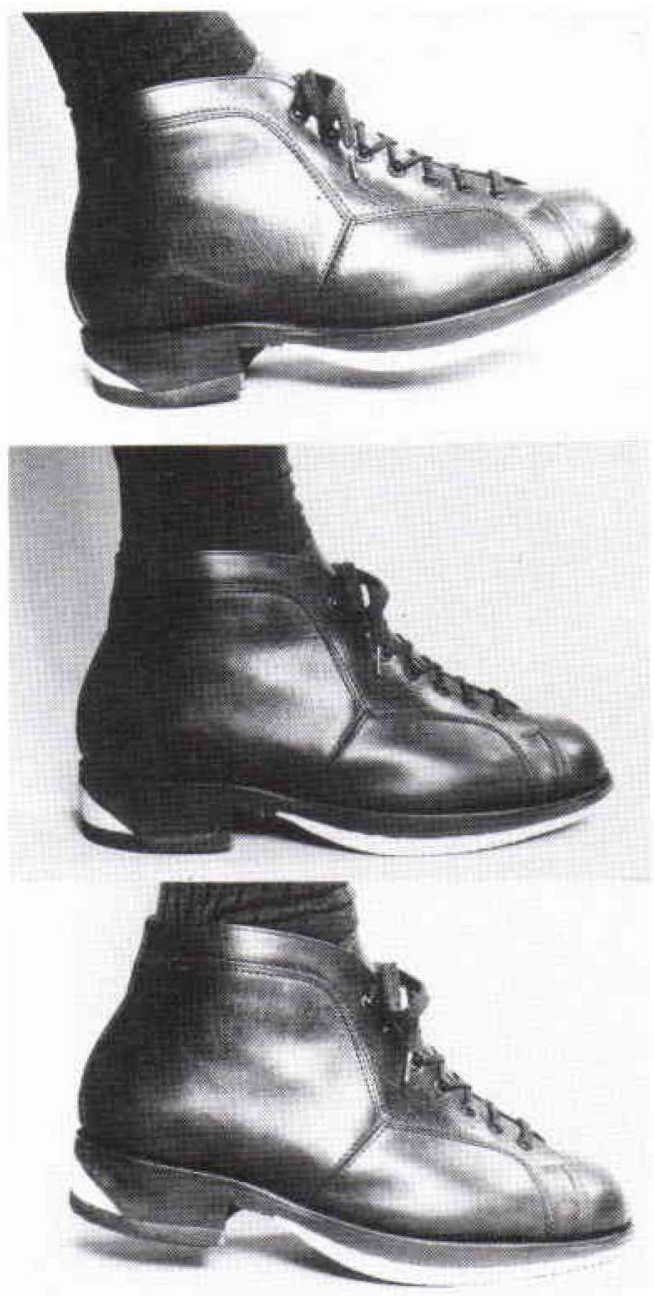

Fig. 4. Example of shoe modification (shown in white). Top, effect of buffer heel on heel-strike. Centre, the rocker sole lifts the forefoot during midstance. Bottom, because of the posteriorly positioned crest of the rocker, less force is exerted during toeoff.

direction of roll-over. However it is also possible to place the crest of the roller on a slant with the lateral side in front of and the medial side behind the metatarsal break. In most cases this arrangement is felt to be more comfortable. Furthermore it is an arrangement which is favoured in the manufacture of insoles. Thus both the insole and the rocker cause increased external rotation of the foot during the rollover.

The bevel of the insole or the crest of the rocker can also be placed towards the other diagonal if internal rotation of the foot during roll-over is desired. However it must be pointed out that this form of rocker or insole is rarely felt to be comfortable and one must be prepared to quickly change the design if it fails to be successful.

\section{High upper}

A high upper is effective in diminishing instability in the "lower ankle joint", thus reducing pain, and enabling the patient to exert greater force. Patients are frequently advised to wear shoes with high uppers. Ready-made sports shoes featuring supplementary reinforcements are especially convenient and can replace expensive custom orthopaedic boots, however good setting of the foot in both frontal and sagittal planes must be achieved; should this not be the case, modifications of these shoes as described above are advised.

\section{Inner shoe}

An inner shoe more effectively immobilizes the "lower ankle joint" but unfortunately strongly inhibits movement at the ankle joint proper (Fig. 5). In the author's opinion short inner shoes with cambered stiffeners barely reaching the ankle are less effective than the ready-made shoes referred to above.

\section{Custom orthopaedic shoe}

This is used for complex sequelae and works basically in the same way as the previously described methods. An orthopaedic shoe can be ordered with e.g. a high stiffener around the heel, horizontal setting of the foot in the sagittal plane, even distribution of weight in the frontal plane, heel buffer and built in rocker. Indeed, it is necessary to require all of these modifications to warrant the manufacture of an orthopaedic shoe. However with bilateral calcaneal fractures it would be sensible to opt for orthopaedic shoes at an earlier stage.

\section{Procedure for mechanical orthopaedic measures after calcaneal fractures}

The initial prescription will include a buffer heel, lowering of the heel, rocker sole and fitting of an insole. Quite possibly these modifications can be applied to low shoes. Primarily the intention is to find out just how severe is the result of the calcaneal fracture. As previously mentioned it is not always possible to anticipate the precise prescription from the type of fracture. Two or three weeks after the 


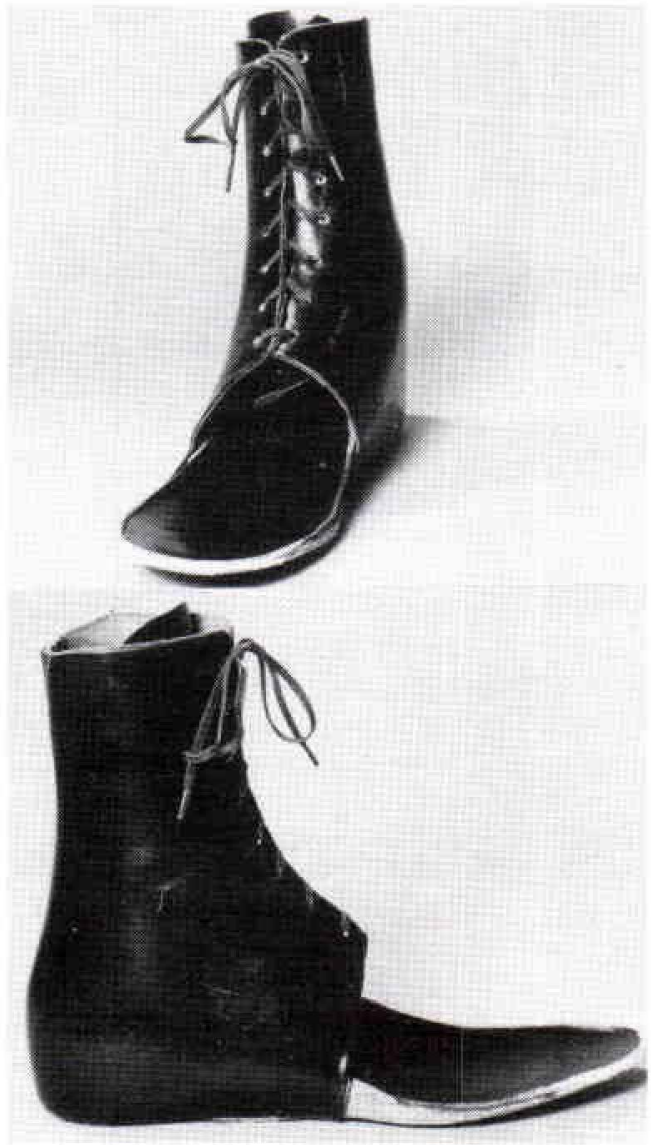

Fig. 5. Example of an inner shoe with full length sole and $20 \mathrm{~cm}$ high upper because of very painful condition. A modified ready-made shoe may be worn over this.

initial fitting the patient returns to the clinic to discuss his complaints.

If severe problems persist shoes with high uppers are strongly recommended. In these, insoles are fitted to gain control of the roll-over as before.

If the symptoms are extremely severe or if immobilization with a high shoe is insufficient, an orthopaedic shoe or inner shoe remains as the last resort. At this point however a surgical procedure such as triple arthrodesis of the talocalcaneal, talonavicular and calcaneocuboid joints should be considered as an alternative. Caution must be exercised as results from this procedure after calcaneal fracture are variable. Hence one will generally continue with mechanical orthopaedic measures and during follow-up with more information available a decision can be made with more confidence.

\section{Conclusion}

In the treatment of calcaneal fractures mechanical orthopaedic measures not only help the patient but also the physician. The patient's concerns are clear: he needs more security and a stronger push-off and must be relieved of pain. The physician can discern significant functional or morphological problems by trial and error. For example he will be in a better position to decide whether a classic triple arthrodesis of the subtalar, talonavicular and calcaneocuboid joints is necessary or if a simpler talocalcanean arthrodesis would suffice. Discussion with the patient and his reaction to the modifications and aids provides information as to how the patient is responding to the injury and the degree of motivation. Thus technical orthopaedic measures are exceedingly valuable elements of comprehensive patient care.

\section{REFERENCES}

Bóhler L. (1977). Die Technik der Knochenbruchbehandlung. 12. Auflage, II. Band, 1. und 2. Teil Wien; Mandrich.

Essex-Lopresti, P. (1952). The mechanism, reduction technique, and results in fractures of the os calcis. Br. J. Surg., 39, 395-419.

Judet, J., Judet, R. (1954). Traitement des fractures du calcanéum avec disjonction astragalocalcanéenne, Acta Orthop. Belg., 20(3), 284-291.

Mattel, R. (1983). Behandlung und Nachbehandlung von Calcaneusfrakturen, Inaugural-Dissertation zur Erlangung der Doktorwürde der Medizinischen Fakultät der Universität Zürich - Zürich: Universitat Zürich.

RABL, C. R. H. (1975). Orthopädie des Fusses Stuttgart: Ferdinand Enke Verlag.

Rowe, C. R., Sakellarides, H. T., Freeman, P. A., Sorbie, C. (1963). Fractures of the os calcis: a long-term follow-up study of 146 patients. JAMA, 184, $920-923$.

Subotnik, St. I. (1975). Pediatric Sports Medicine. Future Publishing Company.

Vidal, J., Orst, G., Lamolineric, G., Reboul, C. (1983). Traitement des fractures articulaires recentes du calcaneum. La place du traitement chirurgical et l'interet de l'osteosynthese par agrafe-tabouret. Acta Orthop. Belg., 49(6), 711-724. 